\title{
Brain-Derived Neurotrophic Factor Is Critically Involved in Thermal-Experience-Dependent Developmental Plasticity
}

\author{
Adi Katz and Noam Meiri \\ Institute of Animal Science, Agricultural Research Organization, Volcani Center, Bet Dagan 50250, Israel
}

All species exhibit critical period for sensory development, yet very little is known about the molecules involved in the changes in the network wiring that underlies this process. Here the role of brain-derived neurotrophic factor (BDNF) in the critical period of thermal control establishment in chicks was investigated.

Neuroanatomically, the body temperature is balanced by the preoptic anterior hypothalamus ( $\mathrm{PO} / \mathrm{AH})$ and controlled by thermosensitive neurons. Exposure to hot or cold conditions during the critical period of temperature control development causes a plastic change in the ratio between heat- and cold-sensitive cells and can modulate temperature tolerance.

It was found that expression of BDNF mRNA but not of NGF or neurotrophin-3 was induced in the PO/AH of 3-d-old chicks during both heat and cold exposure. The peak of BDNF induction in both heat and cold exposure occurred after $6 \mathrm{~h}$, with, respectively, threefold and sevenfold increases in its mRNA expression.

To prove the concept that BDNF activation is a critical step in thermal-experience-dependent plasticity, BDNF was "knocked down" using antisense. It was found that, when $\mathrm{BDNF}$ in the $\mathrm{PO} / \mathrm{AH}$ was inhibited by $80 \%$ during the third postnatal day, thermal establishment was impaired, and, after 1 week, the chicks' body temperature was reduced by $0.5^{\circ} \mathrm{C}$. Furthermore, later in life, their reaction to thermal challenge was altered, and they exhibited a pronounced reduction in their ability to maintain their body temperature and body weight under harsh conditions. Together, these results prove that BDNF is critically involved in thermal-experience-dependent development.

Key words: antisense; thermal conditioning; hypothalamus; chick; critical period; BDNF

\section{Introduction}

Like in other sensory mechanisms, there is a critical period in thermal sensation and temperature control development. Excessive thermal input during the first postnatal week can modulate responses to thermal exposure later in life (Modrey and Nichelman, 1992; Yahav and McMurtry, 2001; Labunskay and Meiri 2006).

The center for regulation of body temperature control in both avians and mammals is neuroanatomically located in the preoptic/anterior hypothalamus $(\mathrm{PO} / \mathrm{AH})$. This area probably plays the dual function of both monitoring local temperature changes and integrating temperature information from the periphery. In avians, during the period of temperature control establishment, there is a continuous change in the proportions between coldand warm-sensitive cells (Tzschentke and Basta, 2000, 2002).

Very little is known about the biochemical mechanism underlying thermal control establishment. Because thermal exposure during the critical period of temperature control founding causes a change in cellular properties in the $\mathrm{PO} / \mathrm{AH}$, it is reasonable to

Received Nov. 17, 2005; revised Feb. 22, 2006; accepted Feb. 23, 2006.

This work was supported by Egg and Poultry Board of Israel Grant 356-0359-00 and Agricultural Research Organization (Volcani Center, Bet Dagan, Israel) Contribution 475/05. We thank S. Yahav for his invaluable suggestions throughout this project. We are grateful to the chicken farm staff for their dedicated work.

Correspondence should be addressed to Dr. Noam Meiri, Institute of Animal Science, Agricultural Research Organization, Volcani Center, P.0. Box 6, Bet Dagan 50250, Israel. E-mail: meiri@agri.huji.ac.il.

DOI:10.1523/JNEUROSCI.0371-06.2006

Copyright $\odot 2006$ Society for Neuroscience $\quad$ 0270-6474/06/263899-09\$15.00/0 expect that the growth signaling mechanism would be involved. Among the neurotrophic factors, there is a direct correlation between brain-derived neurotrophic factor (BDNF) mRNA levels and memory; in the mouse hippocampus, BDNF mRNA was found to be elevated after water maze training (Kesslak et al., 1998), and, in rats, it was higher in the dentate gyrus of good learners than in that of nonlearners trained for passive avoidance memory (Ma et al., 1998) and was upregulated after radial arm maze training (Tokuyama et al., 2000). Furthermore, BDNF has been directly correlated with the biochemical pathways underlying sensory development in the critical period: it can affect the formation of ocular dominance in the cat visual cortex (Gluske et al., 1996), and transgenic mice overexpressing BDNF exhibited accelerated visual development and early closure of the critical period (Huang et al., 1999). In chicks, there are three known neurotrophic factors: nerve growth factor (NGF), BDNF, and neurotrophin-3 (NT-3). Of these, BDNF plays a pivotal role in the developmental plasticity and has been correlated with passive avoidance learning (Johnston et al., 1999; Johnston and Rose, 2001). In the present study, the expression pattern of neurotrophic factors was monitored during the critical period of temperature control establishment.

It could be argued that there might not be a direct correlation between the induction of gene expression and the physiological role of the gene product in thermotolerance acquisition, but disruption of the enzyme would enable its importance in thermotolerance acquisition to be verified. "Knocking down" a protein 
by using antisense provides the opportunity to induce transient changes in gene expression during physiologically significant time windows (Robinson et al., 1997; Kramer and Cohen, 2004). Antisense has been successfully used to downregulate BDNF expression: BDNF antisense injected into the mouse cerebellum caused a memory retrieval deficit in mice that had been radial arm maze trained (Mizuno et al., 2000), injection of BDNF antisense into the mouse dentate gyrus caused learning deficits in passive avoidance memory (Ma et al., 1998), and BDNF antisense injection caused impaired performance of a passive avoidance task by chicks (Johnston and Rose, 2001). Here, the critical period of thermal control establishment was disrupted with BDNF antisense.

\section{Materials and Methods}

\section{Birds, diets, and environment}

Cobb male chicks were obtained in the first day of their lives from Brown Hatcheries (Hod Hasharon, Israel) and raised in climate-controlled rooms at a temperature of $30^{\circ} \mathrm{C}$, under continuous artificial illumination with ad libitum access to food and water. All experiments were performed according to the guidelines of the European Community Council.

\section{Behavioral procedures}

Heat conditioning. On day 3 of their lives, the test chicks were transferred to $37.5^{\circ} \mathrm{C}$ for $24 \mathrm{~h}$, whereas control birds were left at $30^{\circ} \mathrm{C}$. After $30 \mathrm{~min}$ and 2, 6, 12, and $24 \mathrm{~h}$, eight chicks were weighed, a different group each time, their colonic temperatures were measured $(\sim 3.5 \mathrm{~cm}$ in the colon), and blood samples were obtained for corticosterone level measurements.

Thermal challenge. On day 10, both conditioned and nonconditioned experimental chicks were thermally challenged by exposing them to $37.5^{\circ} \mathrm{C}$ for $12 \mathrm{~h}$, whereas the control, nonconditioned chicks were left at $30^{\circ} \mathrm{C}$. After 2, 6, and $12 \mathrm{~h}$, a subgroup of eight chicks, different birds each time, were weighed, their rectal temperatures were measured, and blood samples were obtained for corticosterone level measurements.

Cold conditioning. On day 3 of their lives, chicks were transferred to $16^{\circ} \mathrm{C}$ for $12 \mathrm{~h}$, whereas control chicks were left at $30^{\circ} \mathrm{C}$. After $30 \mathrm{~min}$ and 2,6 , and $12 \mathrm{~h}$, a different group of eight chicks from each time point were weighed, their colonic temperatures were measured, and blood samples were obtained for the corticosterone levels measurements.

Cold acclimation. During the first $10 \mathrm{~d}$, the chicks' environmental temperature was decreased by $0.4^{\circ} \mathrm{C}$ every $6 \mathrm{~h}$ so that, after $10 \mathrm{~d}$, their environmental temperature was $16^{\circ} \mathrm{C}$.

Feed restriction. The average amount of food a chick ate during heat conditioning was measured by weighing the food during the $24 \mathrm{~h}$ of conditioning. Control chicks that were kept at $30^{\circ} \mathrm{C}$ were given the appropriate proportion of this amount of food every $2 \mathrm{~h}$ and were killed after $12 \mathrm{~h}$.

\section{Tissue collection and $m R N A$ purification}

Chicks were killed 2, 6, 12, and $24 \mathrm{~h}$ during thermal conditioning. For mRNA purification, the anterior hypothalamus was dissected and immersed immediately in RNALater (Ambion, Austin, TX). mRNA was purified using Tri reagent (Molecular Research Center, Cincinnati, OH). For in situ hybridization, whole brains were dissected and fixed in $4 \%$ paraformaldehyde in PBS for $24 \mathrm{~h}$.

\section{Sequencing the chick BDNF reading frame}

The $5^{\prime}$ sequence of BDNF was cloned and sequenced using a $5^{\prime}$ primer from the partially known BDNF sequence of chicken (GenBank accession number M83377), 5' -TGCTGGTTCAAGAGGACTGA-3', and a 3' primer from zebra finch BDNF (GenBank accession number AF255389) 5'-GGCGACAAACCACAATGTTA-3'. The PCR product of 919 bp was subcloned into the pGEM-T Easy Cloning Vector (Promega, Madison, $\mathrm{WI}$ ). The purified plasmid was sequenced at the Weizmann Institute sequencing facility (Rehovot, Israel).

\section{Reverse transcription- $P C R$}

Forward (F) and reverse (R) primers used to identify BDNF, NT-3, and NGF by reverse transcription (RT)-PCR were as follows: NGF, F, 5'-
GGACCAAGAGGACTGCACAT-3'; NGF, R, 5'-GGGGGATAGGAGGATGTTGT-3'; NT-3, F, 5' -ACAGACCTCCTAAGGCAGCA-3'; NT-3, R, 5'-GGATGTCTTGCACTGGGAGT-3'; BDNF, F 5' -ACTGGCGGACACTTTTGAAC-3'; and BDNF, R, 5'-GTTGCACCAGACATGTCCAC- $3^{\prime}$. The lengths of the expected amplification products for the NGF, NT-3, and BDNF were 407, 393, and $311 \mathrm{bp}$, respectively. To monitor the integrity of the RNA and to account for variations between tubes that might arise during the experimental manipulations, RT-PCR was conducted using the same samples with primers for chicken $18 \mathrm{~S}$ (expected product, 398 bp): 18S, F, 5' - AGTTGGTGGAGCGATTTGTC3'; and 18S, R, 5'-CCGAGGACCTCACTAAACCA-3'. Hypothalamic RNA was reverse transcribed to single-stranded cDNAs using reverse primers and Moloney murine leukemia virus RT (Invitrogen, Carlsbad, CA) and amplified by PCR. PCR conditions for all mRNAs were $95^{\circ} \mathrm{C}$ for $30 \mathrm{~s}, 60^{\circ} \mathrm{C}$ for $30 \mathrm{~s}$, and $68^{\circ} \mathrm{C}$ for $1 \mathrm{~min}$. In a preliminary set of experiments, the RT-PCR protocols used were optimized for the number of cycles by performing cycle amplification curves to define the linear range for each mRNA. Amplification products arising from RT-PCR were electrophoresed on a $2 \%$ agarose gel and visualized by ethidium bromide staining.

\section{Real-time PCR}

Real-time PCR was performed on a model 7000 sequence analysis system (Applied Biosystems, Foster City, CA). Quantization was established using the Syber green method. The primers were designed so that there would not be primer dimerization, and the amplification curves of the genes were parallel. The expression was determined by comparing the BDNF concentration with those of $18 \mathrm{~S}$ and glyceraldehyde-3-phosphate dehydrogenase (GAPDH), and those of NT-3 and NGF were compared with the expression of the housekeeping gene 18S. Each time point in the 3-d-old chick PO/AH experiment was an average of three independent experiments, and each experiment was a pool of the $\mathrm{PO} / \mathrm{AH}$ from eight chicks. The intermediate medial hyperstriatum ventrale (IMHV), frontal part of the brain (FB), and 12-d-old chick experiments were a comparison of five individual chicks. Statistical analysis of the results was performed with a single-tail ANOVA in which each time point was compared with the expression in naive untreated chicks. The primers used for real-time PCR were as follows: NT-3, right, 5' -CCTCGGTGGCTCTTGTGTT-3'; NT-3, left, 5'-CCACCACCACTGTACCTCA-3'; NGF, right, 5'-TGTTCCATCTGGCATCCAAG-3'; and NGF, left, 5' ACACAGGCAGCTCCAAAGTC-3' The primers used for real-time PCR of BDNF were the same primers used for RT-PCR, and the primers used for GAPDH and $18 \mathrm{~S}$ were as follows: 18S, F, 5'-CGGGTTGGTTTTGGTCTGAT-3'; 18S, R, 5'-ATGGTTCCTTTGGTCGCTCC-3'; GAPDH,

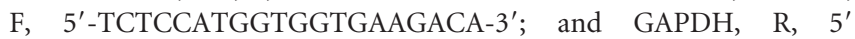
GACGTGCAGCAGGAACACTA-3'.

\section{In situ hybridization}

The brains were removed and fixed in $4 \%$ paraformaldehyde and PBS, $\mathrm{pH} 7$, for $24 \mathrm{~h}$. Then the brains were dehydrated (twice in PBS for $30 \mathrm{~min}$ each and serial alcohol solutions at 50,70, 85, 96, and 100\%), dilapidated in chloroform, and paraffinized at $60^{\circ} \mathrm{C}$. The brains in paraffin were cut into $5 \mu \mathrm{m}$ slices on a microtome. The oligonucleotides designed to detect transcripts encoding sense and antisense BDNF were produced as RTPCR product primers: F, 5' -GGCGACAAACCACAATGTTA-3'; and R, 5'-TGCTGGTTCAAGAGGACTGA-3'. The PCR product was inserted into pGEM-T Easy cloning vector and was used as a template for probe synthesis. The sense and antisense probes were synthesized by in vitro transcription with T7 or SP6 RNA polymerase labeled with $\left[{ }^{35} \mathrm{~S}\right] \mathrm{dUTP}$. The plasmid was sequenced to verify its specificity and to determine the orientation of the insert. The sections were hybridized overnight at $55^{\circ} \mathrm{C}$ in a buffer containing the probe $\left(2-4 \times 10^{7} \mathrm{dpm} / \mathrm{ml}\right)$. The hybridized sections were embedded in emulsion and developed after $30 \mathrm{~d}$. The sections were stained with methyl green counterstain. The hybridization signals for BDNF were evaluated with a computer-assisted image analysis system with both bright- and dark-field microscopy.

\section{Antisense design and injection}

Antisense oligodeoxyribonucleotide (ODN), 5'-AAGGAAAAGGATGGTCAT-3', was designed to hybridize to the AUG translation initiation 
codon of the mRNA encoding BDNF. Neither the antisense nor the sense ODN (5'-ATGACCATCCTTTTCCTT- $\left.{ }^{\prime}\right)$ were expected to hybridize to the mRNA of any other sequence in GenBank except the BDNF sequence. The oligonucleotides were protected by double phosphorothiolation on both the $3^{\prime}$ and 5' ends and were purified by HPLC (Sigma-Genosys, St. Louis, MO). The ODNs were dissolved in saline to a final concentration of $1 \mu \mathrm{g} / \mu \mathrm{l}$.

Injection was performed according to Johnston and Rose (2001), with slight modifications. In brief, a 28 gauge needle was connected via polyethylene PE20 tubing to a Hamilton microsyringe. The injection was into the third ventricle area, with coordinates $6 \mathrm{~mm}$ anterior from the ear position in the middle of the skull (coordinates according to Kuenzel and Masson, 1988). The injection needle was fitted with a stop that limited penetration to a maximum of $6 \mathrm{~mm}$. The injection location was verified in preliminary experiments. Because the chicks have a soft unossified skull, this procedure does not require anesthetic and is routinely performed without administration of analgesics (Johnston and Rose, 2001). The chicks were not overly distressed by the injection, and their behavior seemed normal.

\section{Antisense pharmacokinetics}

The effectiveness of antisense inhibition was determined by two procedures. In the first, $2 \mu \mathrm{g}$ of antisense was injected, and the amount of $\mathrm{BDNF}$ in the PO/AH was evaluated $1,2,6,12$, and $24 \mathrm{~h}$ after injection by semiquantitative RT-PCR and by real-time PCR. In the second, an increasing series of antisense doses $(0.5,1,2$, and $5 \mu \mathrm{g})$ were injected, and the degree of inhibition in the $\mathrm{PO} / \mathrm{AH}$ was evaluated $6 \mathrm{~h}$ after injection by both RT-PCR and real-time PCR. Control chicks were injected with either the $2 \mu \mathrm{g}$ of sense or with $2 \mu \mathrm{l}$ of $0.9 \% \mathrm{NaCl}$ solution. In addition, both the IMHV and the frontal $2 \mathrm{~mm}$ of the brain were dissected from each chick to control for the amount of nonspecific BDNF inhibition in parts of the brain that were not targeted.

\section{Behavioral effect of antisense inhibition}

The changes in body weight and body temperature of chicks in the four groups ( $n=8$ in each group) were evaluated during exposure to $37.5^{\circ} \mathrm{C}$ on day 10 of their lives. Two of the groups had been injected on day 3 , one with sense and the other with antisense, and then kept in a constant temperature of $30^{\circ} \mathrm{C}$; the other two groups had been injected with either sense or antisense on day 3 , exposed immediately after injection to $37.5^{\circ} \mathrm{C}$ for $24 \mathrm{~h}$, and returned to their home cage at $30^{\circ} \mathrm{C}$ until day 10 .

\section{Results}

\section{The expression of BDNF but not of NGF or NT-3 was induced during heat conditioning}

Because the working hypothesis was that plastic events during the critical period of temperature control establishment in the hypothalamus are responsible for the long-term changes in the temperature control set point, changes in the expression of neurotrophic factors were monitored during the time window known to be most effective for heat conditioning in chicks (i.e., postnatal day 3). Several molecular methods were used, each applied to a different behavioral experiment. The expression of three neurotrophic factors, BDNF, NGF, and NT-3, was monitored, and it was established that the mRNA of BDNF but not those of NGF or NT-3 was induced during conditioning. The expression pattern of BDNF, as revealed by RT-PCR amplification with specific primers between 3 and $24 \mathrm{~h}$ after the start of treatment, indicated that the amplification began $3 \mathrm{~h}$ after the start of conditioning and peaked $6 \mathrm{~h}$ into the treatment (Fig. $1 A$ ). After $24 \mathrm{~h}$, the expression level had declined back to the level observed at $3 \mathrm{~h}$. The expression patterns of two other neurotrophic factors, NGF and NT-3, in the PO/AH in the same conditioned chicks were not altered (Fig. $1 A$ ). To quantify the heat conditioning-related induction of BDNF in the frontal hypothalamus, real-time PCR was used. BDNF expression levels were measured and compared with those of two housekeeping genes, GAPDH and 18S, in three in- dependent experiments. The expression level of BDNF compared with the housekeeping genes was elevated by $1.95 \pm 0.452 \mathrm{~h}$ into conditioning and peaked $6 \mathrm{~h}$ into thermal conditioning, at which time the expression level was $3.15 \pm 0.75$ times higher than that of the expression level of BDNF in untreated chicks. After $12 \mathrm{~h}$, the expression was elevated by $2.8 \pm 0.8$, and, after $24 \mathrm{~h}$, the expression level of BDNF returned to its naive level $(0.95 \pm 0.25)$. The induction of the gene after both 6 and $12 \mathrm{~h}$ was statistically significant (ANOVA, $p<0.05$ ) (Fig. $1 B$ ). Real-time PCR quantitation of the expression of NT-3 and NGF revealed that the expression of the mRNA of these genes did not change as a result of heat conditioning. The expression of NGF during heat conditioning compared with the expression of NGF in naive chicks was $1.2 \pm$ 0.1 after $2 \mathrm{~h}, 1.08 \pm 0.21$ after $6 \mathrm{~h}, 1.22 \pm 0.44$ after $12 \mathrm{~h}$, and $1.32 \pm 0.32$ after $24 \mathrm{~h}$. The expression of NT-3 during heat conditioning compared with the expression of NT-3 in naive chicks was $1.02 \pm 0.05$ after $2 \mathrm{~h}, 1.2 \pm 0.2$ after $6 \mathrm{~h}, 0.91 \pm 0.25$ after $12 \mathrm{~h}$, and $1.03 \pm 0.08$ after $24 \mathrm{~h}$ (Fig. $1 B$ ).

A proper control for this experiment should dissociate heat conditioning from both hypothalamic activity and general stress. Because the hypothalamus also controls feed consumption and drinking behavior and there is a reduction in the nourishment pattern of chicks during heat conditioning, we used feed restriction as a control and checked whether there was a change in the expression pattern of BDNF in the hypothalamus during feed restriction. Feed restriction serves also as a control for general stress. We found that an average chick consumed $23.75 \mathrm{~g}$ of food during $12 \mathrm{~h}$ of heat conditioning. We supplied that exact amount of food, divided among six equal servings to untreated chicks that were housed under a normal raising temperature, and found that the expression level of BDNF in the hypothalamus in feedrestricted chicks was identical to that in naive chicks. To ensure that the expression difference was not a result of the amplification reaction, we checked the expression pattern of the housekeeping gene $18 \mathrm{~S}$ in the same reverse-transcribed reaction and found that the level of this mRNA did not change with time after conditioning (Fig. 1C). The expression of BDNF in the PO/AH of $12 \mathrm{~h}$ of feed-restricted chicks as measured using real-time PCR was $1.2 \pm$ 0.17 compared with naive chicks (Fig. $1 B, D$ ). These results indicate that neither stress nor a controlled feeding schedule affected BDNF expression. To confirm the discrete neuroanatomical location of the induction in the $\mathrm{PO} / \mathrm{AH}$, the expression of $\mathrm{BDNF}$ was evaluated in two additional brain areas, IMHV and FB, using both RT-PCR and real-time PCR. In these two areas, there was no change in the expression level of BDNF as a result of heat conditioning (Fig. $1 E, F$ ). To correlate the time of expression of BDNF with the critical period of thermal control establishment, the expression of BDNF was evaluated in the $\mathrm{PO} / \mathrm{AH}$ of 12 -d-old chicks that had been exposed to $37.5^{\circ} \mathrm{C}$ for $24 \mathrm{~h}$ using both RT-PCR and real-time PCR, and it was found that, in chicks that had passed the critical age of thermal control establishment, the expression of $\mathrm{BDNF}$ in the $\mathrm{PO} / \mathrm{AH}$ was not altered by heat treatment (Fig. $1 G, H)$.

To further visualize the location of BDNF expression during conditioning, we performed in situ hybridization. As a first stage, to obtain a long probe, the sequence of the reading frame of chick BDNF was completed by using a left primer from the known chick sequence and a right primer from a zebra finch (the GenBank accession number of the reading frame of BDNF obtained was DQ124361). The probe obtained from this amplification product was sequenced and hybridized with sagittal sections from naive and $6 \mathrm{~h}$ heat-conditioned chicks, and, as can be seen in the representative slide, expression was visualized in the $\mathrm{PO} / \mathrm{AH}$ area and in the cere- 
bellum. However, whereas in the cerebellum the expression was unaffected by the thermal conditioning, in the $\mathrm{PO} / \mathrm{AH}$, it was induced $6 \mathrm{~h}$ into conditioning (Fig. $2 A, E$ ). The difference in the background expression that seems stronger in the section from the naive chick was a result of the very profound induction pattern in the heat-induced slice in the PO/AH, which necessitated a slightly shortened exposure (Fig. 2E). To depict better the BDNF expression in the $\mathrm{PO} / \mathrm{AH}$, an enlargements of the $\mathrm{PO} / \mathrm{AH}$ was performed (Fig. $2 B$ ). An additional enlargement of an area within the $\mathrm{PO} / \mathrm{AH}$ is presented using both bright- and dark-field microscopy to demonstrate the regularity of distribution of the BDNF-induced cells in this area (Fig. $2 C, D)$. As can be seen in the bottom panel, the sense hybridization did not yield any nonspecific mRNA signal (Fig. $2 F$ ).

\section{The expression of BDNF but not of NGF or NT-3 is induced during cold conditioning}

Because hot and cold conditioning are probably mediated by different sets of cells but both processes nevertheless are performed in the $\mathrm{PO} / \mathrm{AH}$, we wanted to check whether BDNF was also involved in the biochemical pathways that underlie cold conditioning. Furthermore, a change in thermal adaptation can be achieved by two mechanisms: acclimation, which uses a slow change in environmental temperature, and conditioning, which uses extreme temperature exposure during the critical period of temperature control establishment. In the present study, after we had established that BDNF expression was correlated with thermal conditioning, our second goal was to check its expression pattern as related to acclimation. Therefore, BDNF expression pattern was evaluated after cold acclimation.

The chicks were cold conditioned on day 3 of their lives by exposing them to $16^{\circ} \mathrm{C}$. In contrast to conditioning, which is a relatively short process $(24 \mathrm{~h})$, effective acclimation takes several days to enable the temperature of the conditioning process to be matched with that of the acclimation process. We acclimated chicks by reducing their environmental temperature by $0.4^{\circ} \mathrm{C}$ every $6 \mathrm{~h}$ so that, by the end of the process (after $9 \mathrm{~d}$ ), the environmental temperature was $16^{\circ} \mathrm{C}$.

The expression pattern of BDNF in the $\mathrm{PO} / \mathrm{AH}$ during cold conditioning and after cold acclimation, as examined by means of several molecular techniques, revealed that the gene was induced during conditioning but that there was no change in its expression level after acclimation. In addition, there was no change in the expression levels of NT-3 and NGF as a result of both cold conditioning and acclimation (Fig. $3 A, B$ ).

We first examined the expression pattern by means of the
A.

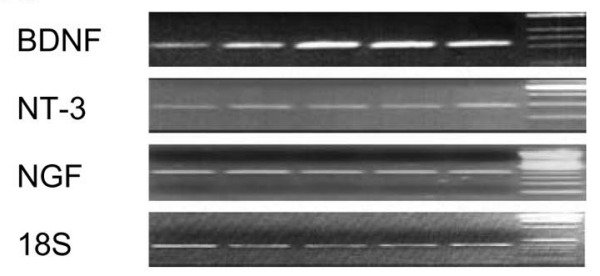

B.

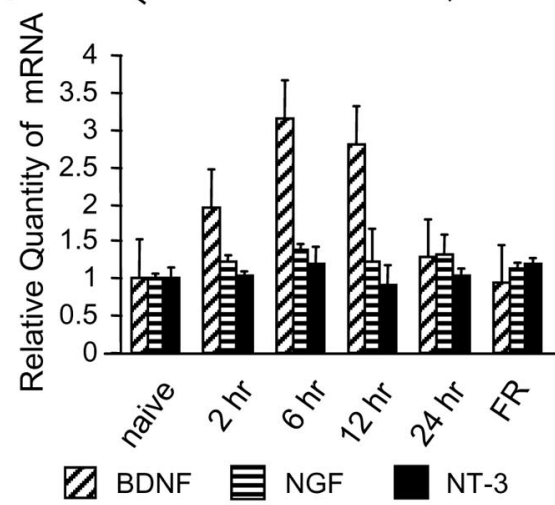

C.

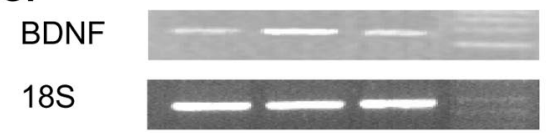

D.

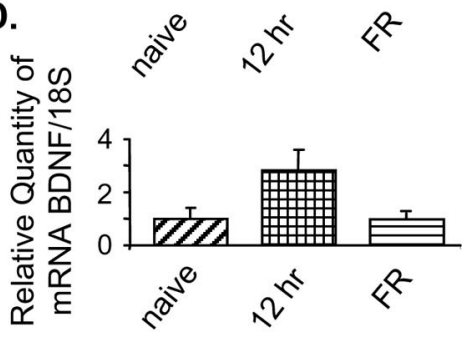

E.

\section{IMHV}

FB

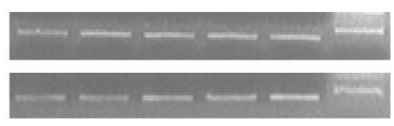

F.

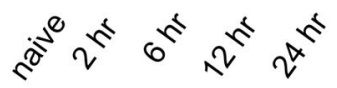

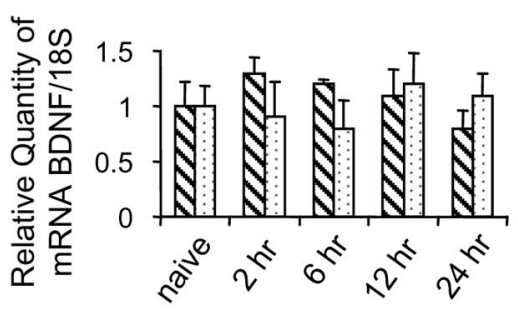

IMHV $\mathbf{N}$ FB
G.

BDNF

$18 S$

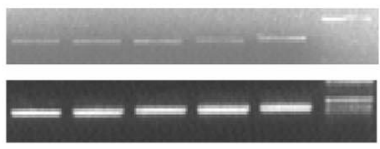

H.
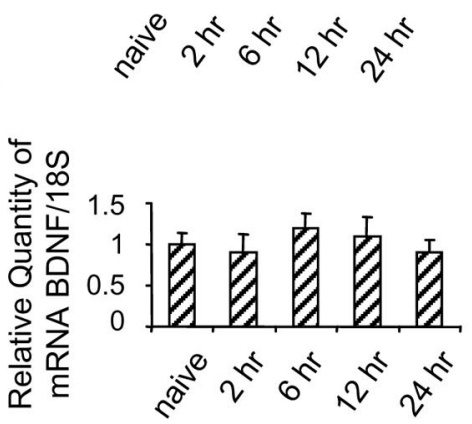

Figure 1. Alteration of BDNF mRNA levels in the chick PO/AH during heat conditioning. A, RT-PCR with specific BDNF primers; total RNA extracted from chicks' $\mathrm{PO} / \mathrm{AH}$ at 3, 6, 12, and $24 \mathrm{~h}$ into conditioning. Each data point in this gel represents a pool of eight chicks. The same samples were amplified with primers specific to NGF and NT-3 and with primers specific to the housekeeping gene 18S. B, Real-time PCR quantitation; the BDNF expression level was compared with that of both 185 and GAPDH. The NGF and NT-3 expression was compared with the expression of $18 \mathrm{~S}$ using the Syber green method. Each time point is an average of three independent experiments, and each experiment is a pool of the PO/AH from eight chicks. All of the results presented in the figure are average \pm SEM.C, RT-PCR with BDNF-specific primers, applied to $m$ RNA from PO/AH from naive chicks and from those that had undergone $12 \mathrm{~h}$ of either heat conditioning or feed restriction. The same samples were amplified with primers specific to the housekeeping gene $18 \mathrm{~S}$. Each data point in these gels represents a pool of eight chicks. $\boldsymbol{D}$, Real-time PCR quantitation of the samples described in $\boldsymbol{C}$ using the Syber green method. The BDNF expression level was compared with that of 18S. E, RT-PCR amplification with BDNF-specific primers of samples from the IMHV and FB during heat conditioning. $\boldsymbol{F}$, Real-time PCR quantitation of the samples described in $\boldsymbol{E}$ using the Syber green method. BDNF expression level was compared with that of 185 . The expression level in each time point was compared with the expression level in naive chicks in the relevant brain area. G, RT-PCR amplification with BDNF-specific primers of samples from the P0/AH of 12-d-old chicks after heat exposure. The same samples were amplified with primers specific to the housekeeping gene $18 S$. $\boldsymbol{H}$, Real-time PCR quantitation of the samples described in $\boldsymbol{G}$ using the Syber green method. BDNF expression level was compared with those of 185 . The expression level in each time point was compared with the expression level in naive chicks.

semiquantitative RT-PCR method with specific primers, which we applied at intervals between $20 \mathrm{~min}$ and $12 \mathrm{~h}$ into conditioning. The findings indicated that BDNF induction peaked $6 \mathrm{~h}$ into conditioning (Fig. $3 A$ ) and that the expression level declined back to its naive level by $12 \mathrm{~h}$ into conditioning. There was no difference between the expression level of BDNF in naive chicks that were held in an environmental temperature of $30^{\circ} \mathrm{C}$ and that in 

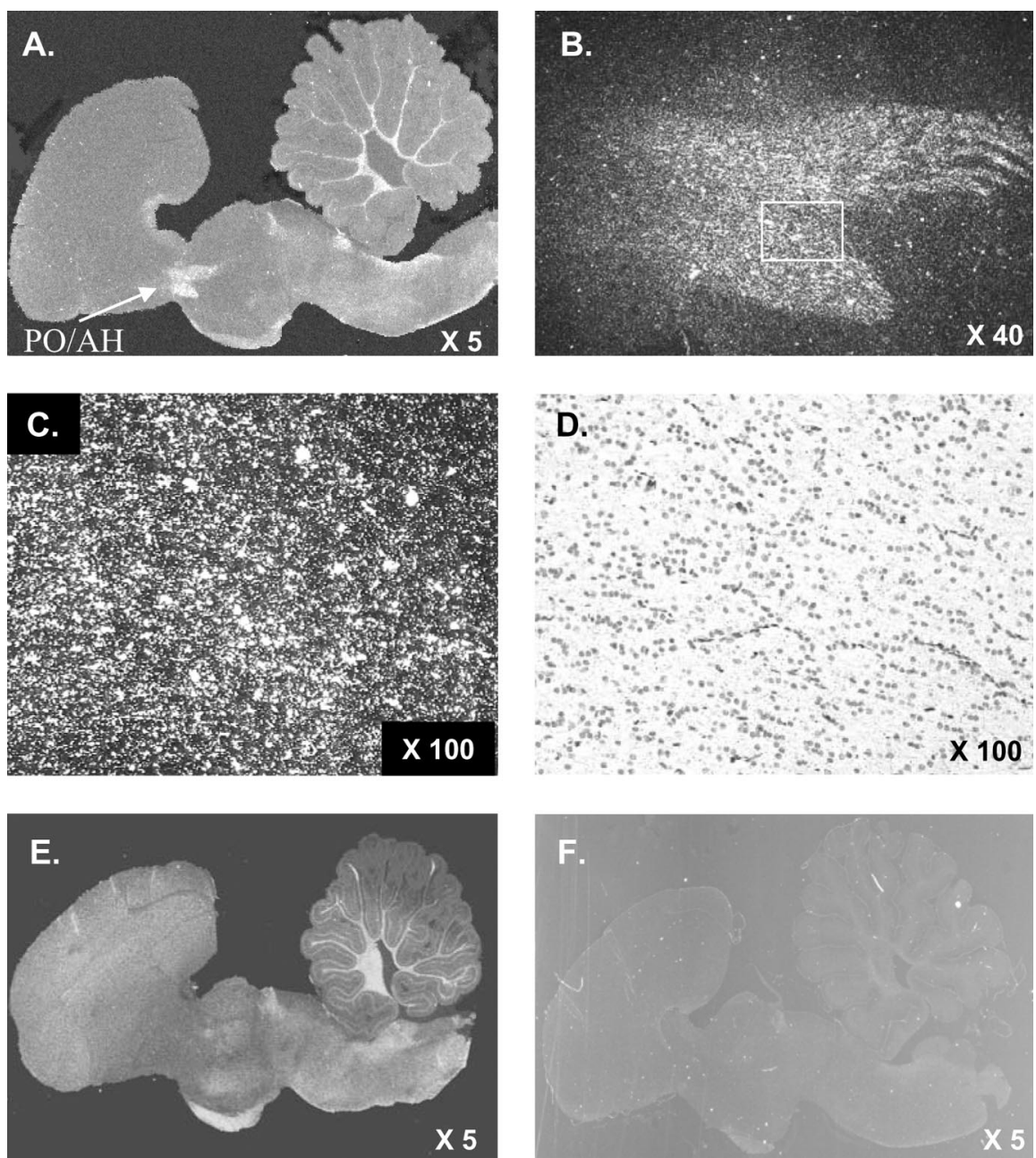

Figure 2. A representative in situ hybridization of sagittal sections hybridized with a BDNF probe. In situ hybridization was performed with a radiolabeled probe and visualized with photographic emulsion. The sections are sagittal sections, with coordinates according to Kuenzel and Masson (1988). Lateral, $0.5 \mathrm{~mm}$. $\boldsymbol{A}$, Six hours into heat conditioning. $\boldsymbol{B}$, An enlargement $(40 \times)$ of the PO/AH from a $6 \mathrm{~h}$ conditioned chick. $C$, An enlargement $(100 \times)$ of the field of the $\mathrm{PO} / \mathrm{AH}$, which is marked with a rectangle in B. D, A bright-field enlargement $(100 \times)$ of the same area depicted in $\boldsymbol{C}$ counterstained with methyl green. $\boldsymbol{E}$, Naive age-matched, nonconditioned chicks. $\boldsymbol{F}$, Sense-treated brain section.

those that had undergone $10 \mathrm{~d}$ of acclimation during which they were held at $16^{\circ} \mathrm{C}$. Similar to the expression pattern observed during heat conditioning, the induction of BDNF in cold conditioning was specific, as indicated by the finding that the expression levels of NGF and NT-3 were not altered. In addition, to ensure that the expression difference was not a result of the amplification reaction, we checked the expression pattern of the housekeeping gene $18 \mathrm{~S}$, which was evaluated in the same reversetranscribed reaction, and found that the level of this mRNA did not change with time after conditioning (Fig. $3 A$ ).

To quantify the cold-conditioning-related induction of BDNF in the frontal hypothalamus, real-time PCR was used. The expression levels of BDNF, NGF, and NT-3 were measured and compared with those of $18 \mathrm{~S}$ in three independent experiments. The expression level of BDNF compared with the housekeeping genes peaked $6 \mathrm{~h}$ into thermal conditioning, at which time the expression level was $6.4 \pm 1.2$ times higher than that of the expression level of BDNF in untreated chicks, and, after $12 \mathrm{~h}$, the expression level of BDNF returned to its naive level $(1.3 \pm 0.24)$. The induction of the gene after $6 \mathrm{~h}$ was statistically significant (ANOVA, $p<0.05$ ) (Fig. 3B). Real-time PCR quantitation of the expression of NT-3 and NGF revealed that the expression of the mRNA of these genes did not change as a result of cold conditioning. The expression of NGF during cold conditioning compared with the expression of NGF in naive chicks was $0.9 \pm$ 0.23 after $20 \mathrm{~min}, 1.2 \pm 0.34$ after $2 \mathrm{~h}$, $1.1 \pm 0.24$ after $6 \mathrm{~h}$, and $0.8 \pm 0.19$ after $12 \mathrm{~h}$. The expression of NT-3 during cold conditioning compared with the expression of NT-3 in naive chicks was $1.1 \pm$ 0.23 after $20 \mathrm{~min}, 1.4 \pm 0.25$ after $2 \mathrm{~h}$, $1.2 \pm 0.6$ after $6 \mathrm{~h}$; and $0.92 \pm 0.3 \mathrm{after}$ $12 \mathrm{~h}$ (Fig. 3B).

As in the heat conditioning experiment, to confirm the discrete neuroanatomical location of the induction in the $\mathrm{PO} / \mathrm{AH}$, the expression of BDNF was evaluated in two additional brain areas, IMHV and FB, using both RT-PCR and real-time PCR. In these two areas, there was no change in the expression level of BDNF as a result of cold conditioning (Fig. 3C,D).

\section{Antisense knock-down of BDNF} impairs thermal control establishment To establish the physiological role of a protein, it is not enough to show that it is induced by temperature exposure during the critical period; it is eminent to show that dysfunction of the protein impairs the establishment of temperature control. Therefore BDNF was knocked down by an intracranial antisense injection. The antisense sequence used was previously used successfully to inhibit BDNF in the chick IMHV (Johnston and Rose, 2001). Nevertheless, to use it for inhibiting the expression of BDNF in the developmental stage of thermal control establishment, the pharmacokinetic parameters for this inhibitor were determined in the $\mathrm{PO} / \mathrm{AH}$. The optimal dose was established by injection of a series of antisense concentrations ranging between 0.5 and $2 \mu \mathrm{g}$, and it was found that the inhibition increased in a dose-dependent manner. Compared with the expression levels of the PO/AH BDNF in chicks injected with saline or $2 \mu \mathrm{g}$ of sense, a $0.5 \mu \mathrm{g}$ dose of antisense induced inhibition of 55\%, $1 \mu \mathrm{g}$ inhibited by $\sim 85 \%$, and $2 \mu \mathrm{g}$ inhibited by $93 \%$ (Fig. $4 A, B$ ). The duration of inhibition was determined by measuring the BDNF mRNA expression level between 2 and $48 \mathrm{~h}$ after injection. It was found that inhibition started $2 \mathrm{~h}$ after injection, at which time there was a reduction of $20 \%$, and that the maximum inhibition, by $\sim 80 \%$, occurred $6 \mathrm{~h}$ after injection. After $12 \mathrm{~h}$, there was 35\% inhibition, and inhibition lasted until $24 \mathrm{~h}$ after injection, at which time there was a $25 \%$ reduction in the BDNF mRNA expression. Forty-eight hours after injection, there was a slight $10 \%$ increase in the amount of BDNF mRNA in the PO/AH (Fig. $4 C, D$ ). To check the spatial extent of the inhibition within the brain, the BDNF expression level after injection was determined in two additional brain areas: IMHV, which was implicated with the critical period of filial imprinting (Matsushima et al., 2003), and FB. Measuring the expression level of BDNF in the IMHV using real-time PCR 
revealed that there was a slight $21 \%$ reduction in BDNF expression $6 \mathrm{~h}$ after administration of the inhibitor $(0.78 \pm 0.14)$, whereas in the $\mathrm{FB}$, the levels of BDNF after antisense injection were unaffected (Fig. $4 E, F$ ).

After the effectiveness of BDNF inhibition through the use of antisense had been confirmed, the phenotypic effect of BDNF knock-down was evaluated by inhibiting BDNF expression on postnatal day 3 while exposing the chicks to various environmental temperatures for $24 \mathrm{~h}$. Because the aim of this experiment was to evaluate the long-term effects of BDNF inhibition, the chicks' thermal response was monitored after 1 week (Fig. 5A). It was found that antisense inhibition of BDNF during postnatal day 3 (i.e., during the critical period of temperature control establishment), significantly reduced the chicks' body temperature 1 week later in an environmental temperature of $30^{\circ} \mathrm{C}$ : whereas the body temperatures of chicks that were injected with the antisense sequence and exposed to either 30 or $37.5^{\circ} \mathrm{C}$ were $41.0 \pm 0.05$ and $41.1 \pm 0.1^{\circ} \mathrm{C}$, respectively, those of chicks that were injected with the sense sequence and raised at 30 or $37.5^{\circ} \mathrm{C}$ were $41.6 \pm 0.08$ or $41.5 \pm 0.08^{\circ} \mathrm{C}$, respectively (ANOVA, $p<0.01 ; n=8$ in each group) (Fig. $5 B$ ).

To evaluate the long-term effect of the lack of BDNF during temperature control establishment, four groups of chicks were heat challenged by exposing them to $37.5^{\circ} \mathrm{C}$ on the day 10 of their lives, long after the antisense seized to affect. Two of the groups had been injected on day 3 , one with sense and the other with antisense, and then kept at a constant temperature of $30^{\circ} \mathrm{C}$; the other two groups had also been injected with either sense or antisense on day 3 but they were then immediately exposed to heat conditioning at $37.5^{\circ} \mathrm{C}$ for $24 \mathrm{~h}$ (Fig. $5 A$ ). BDNF knockdown during the establishment of temperature control caused a significant reduction in the chicks' ability to maintain their body temperature when exposed to an extreme environmental temperature later in life (Fig. 5C). One hour after the beginning of heat challenge on day 10 of life, the body temperature of chicks that were antisense injected and heat conditioned on day 3 had risen by $2.65 \pm 0.1^{\circ} \mathrm{C}$, and, after $3 \mathrm{~h}$, it had risen significantly by an additional $0.65^{\circ} \mathrm{C}$ (i.e., a total rise of $3.30 \pm 0.23^{\circ} \mathrm{C}$ ) (ANOVA, $p<0.01$ ). After $12 \mathrm{~h}$, their body temperature returned to the level recorded $1 \mathrm{~h}$ after the beginning of conditioning (i.e., a rise of $2.62 \pm 0.2^{\circ} \mathrm{C}$ ). In contrast, the body temperatures of senseinjected chicks that were either raised at a normal temperature $\left(30^{\circ} \mathrm{C}\right)$ or heat conditioned on day 3 (at $37.5^{\circ} \mathrm{C}$ ) had risen by only $\sim 1^{\circ} \mathrm{C} 1 \mathrm{~h}$ after the beginning of heat challenge and stayed constant throughout the $12 \mathrm{~h}$ during which the chicks were exposed to extreme heat.

Because the critical period of sensory development depends on the amount of sensory input, the effect of BDNF inhibition was evaluated by exposing chicks to different temperatures. It was found that, under BDNF inhibition, there was a significant long-term difference in temperature control development between chicks that were exposed during development to 30 or $37.5^{\circ} \mathrm{C}$. Whereas the body temperature of antisenseinjected chicks that were heat exposed during the critical period level was compared with that of 185 .
A.

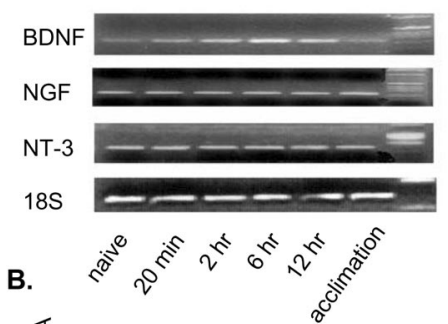

C.

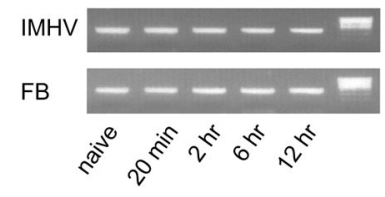

D.
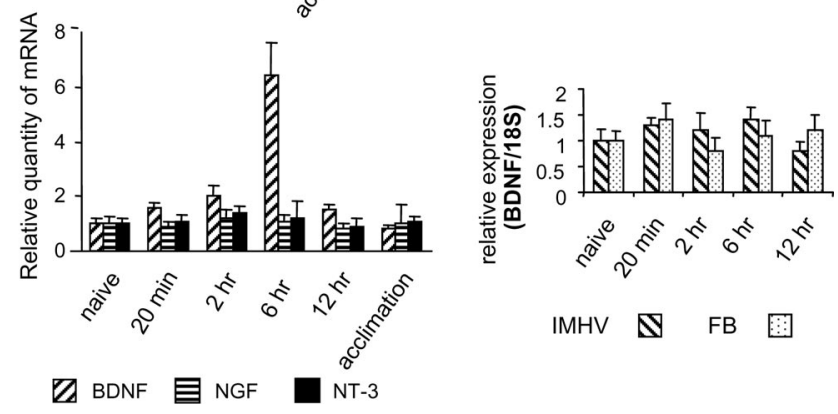

IMHV $\mathbb{F B}$

Figure 3. Alteration of BDNF mRNA levels in chick $\mathrm{PO} / \mathrm{AH}$ during cold conditioning and acclimation. A, RT-PCR with BDNF-specific primers; total RNA extracted from chick PO/AH in time course 20 min and 2, 6, and $12 \mathrm{~h}$ into conditioning. Each data point in this panel and in the rest of this figure represents a pool of eight chicks. The same samples amplified with primers specific to NGF and NT-3 and with primers specific to the housekeeping gene 185 . $\boldsymbol{B}$, Real-time PCR quantitation; the BDNF, NGF, and NT-3 expression was compared with the expression of 185 using the Syber green method. Each time point is an average of three independent experiments, and each experiment is a pool of the PO/AH from eight chicks. All of the results presented are average \pm SEM. C, RT-PCR amplification with BDNF-specific primers of samples from coldexposed 3-d-old chicks. mRNA was extracted from the IMHV and from the FB of five chicks and was pooled. The same samples were amplified with primers specific to the housekeeping gene 18S. D, Real-time PCR quantitation of the samples described in Cusing the Syber green method. BDNF expression level was compared with that of $18 \mathrm{~S}$.

A.
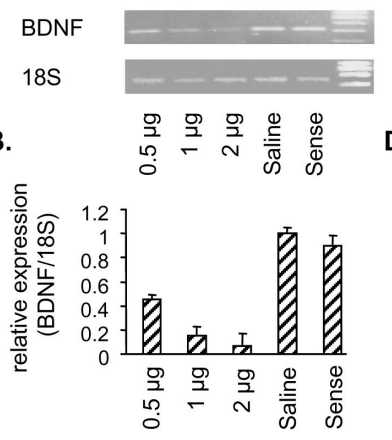

C.

BDNF
$18 \mathrm{~S}$
D.
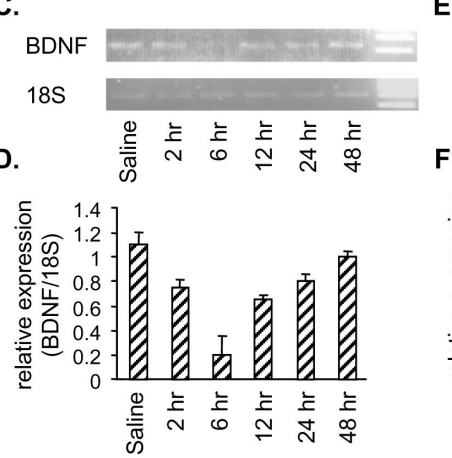

E.
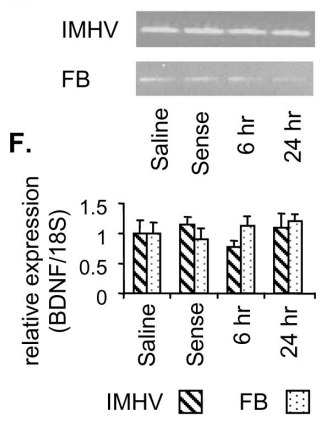

Figure 4. Pharmacokinetic evaluation of antisense inhibition of $\mathrm{BDNF}$ in the $\mathrm{PO} / \mathrm{AH}$ of 3 -d-old chicks. $A, \mathrm{RT}-\mathrm{PCR}$ analysis of the effects of different antisense doses on BDNF expression in the P0/AH of 3-d-old chicks: $0.5,1$, and $2 \mu \mathrm{g}$ of the BDNF-specific antisense or sense sequence or of saline were injected into the third ventricle. Each lane represents a pool of mRNA from the PO/AH of four injected chicks $6 \mathrm{~h}$ after injection. The same samples were amplified with primers specific to the housekeeping gene 185 . $\boldsymbol{B}$, Real-time PCR quantitation of the antisense dose effect. Comparison of the expression of BDNF with 18S. C, RT-PCR analysis of the effective antisense BDNF inhibition time in the P0/AH of 3-d-old chicks. Each lane on the gel represents the mRNA from a pool of $\mathrm{PO} / \mathrm{AH}$ from four chicks injected in the third ventricle with BDNF-specific antisense and killed 2,6, 12, 24, and $48 \mathrm{~h}$ after injection. The sense represents the effect $6 \mathrm{~h}$ after injection. $\boldsymbol{D}$, Real-time quantitation of the time dependency of antisense inhibition. Comparison of the expression of BDNF with that of $18 S . E$, RT-PCR amplification with BDNF-specific primers of samples from the IMHV and FB of chicks injected in the third ventricle with antisense to BDNF for 6 or $24 \mathrm{~h}$ after injection or with sense or saline for $6 \mathrm{~h}$ after injection. $\boldsymbol{F}$, Real-time PCR quantitation of the samples described in $\boldsymbol{E}$ using the Syber green method. BDNF expression 
A.

C.

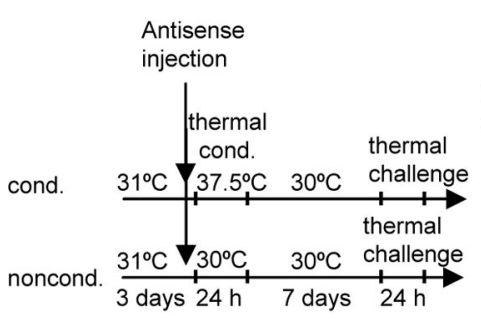

B.

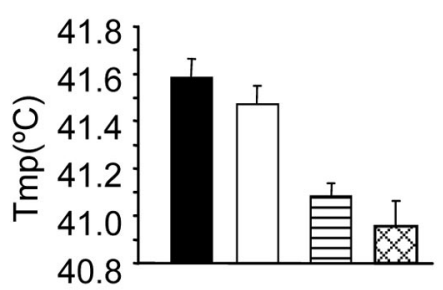

cond. sense
noncond. sense
cond. antisense
noncond. antisense

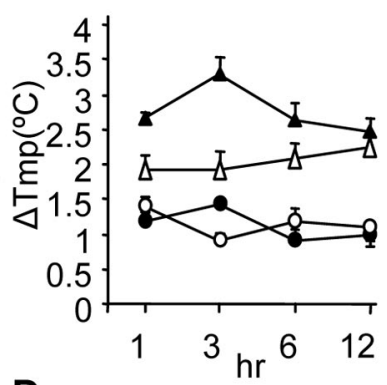

D.
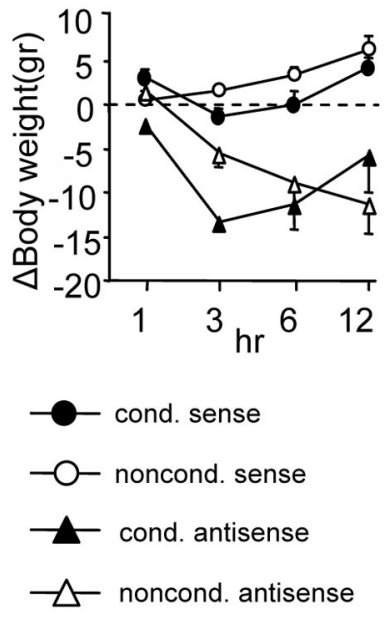

$37.5^{\circ} \mathrm{C}$ for $24 \mathrm{~h}$. The changes in the body weights of the chicks in each of the groups were different (i.e., conditioning caused a significant change in the body weight regimen). Whereas the body weights of sense-injected chicks that had not been conditioned during development rose steadily under exposure to extreme temperature (their body weight rose by $1.14 \pm 1 \mathrm{~g}$ after $1 \mathrm{~h}$, by $2.14 \pm 0.55 \mathrm{~g}$ after $3 \mathrm{~h}$, by $4 \pm 0.82 \mathrm{~g}$ after $6 \mathrm{~h}$, and by $7 \pm 1.38 \mathrm{~g}$ after $12 \mathrm{~h}$ ), the body weights of conditioned chicks rose by $3.86 \pm$ $0.88 \mathrm{~g}$ in the first hour but then fell by $0.71 \pm 1.02 \mathrm{~g}$ after $3 \mathrm{~h}$, and their weight gain resumed only after $6 \mathrm{~h}$ (multivariate ANOVA, i.e., ANOVA with repeated measures, $p<0.01 ; n=8$ in each group).

In contrast to sense-injected chicks, which gained weight during heat challenge, antisense-injected chicks, both conditioned and nonconditioned, lost weight during challenge. However, whereas conditioned chicks lost most of their weight in the first $3 \mathrm{~h}$ of conditioning (loss of $12 \pm 0.6 \mathrm{~g}$ ) but recovered and started gaining weight later so that after $12 \mathrm{~h}$ they had lost only $5 \pm 4.28 \mathrm{~g}$, the nonconditioned chicks gained $2.29 \pm 1.36 \mathrm{~g}$ in the first hour but, from then to the end of the experiment, they lost weight steadily, for a total loss of $10.57 \pm 3.41 \mathrm{~g}$ during the $12 \mathrm{~h}$ of measurements.

\section{Discussion}

Previous studies have demonstrated that thermal conditioning induces long-term changes in the chicks' ability to withstand heat stress later in life (Horowitz, 1998; Yahav and McMurtry, 2001; Labunskay and Meiri, 2006). Furthermore, in young precocial birds, during development, there is a shift in the ratio between cold- and warm-sensitive cells (Tzschentke and Basta, 2000, 2002). In the present study, we demonstrated that BDNF plays a critical role in these long-term changes. It was found that BDNF was induced in the PO/AH during both hot and cold conditioning, with a peak $6 \mathrm{~h}$ into conditioning, and that, when BDNF was knocked down by means of injected antisense, with an inhibition peak $6 \mathrm{~h}$ into conditioning, the establishment of thermal control was impaired.

Although all species, ranging from Drosophila to humans, exhibit critical periods for sensory development, very little is known about the molecules involved in the network wiring that underlies this process (Hensch, 2004). The domestic chick is an optimal model system to study sensory development of both prenatal and postnatal critical periods because these birds are able to learn immediately after hatching and, more important, their behavioral repertoire after hatching can be measured easily (Matsushima et al., 2003). The proteins that have been implicated in network organization in the critical period of sensory development are the NMDA receptor, neurotrophins, and the GABAergic systems (Berardi et al., 2000; Boettiger and Doupe, 2001). Similar to the present findings that indicate the pivotal role of BDNF in the critical period of thermal control establishment, BDNF has been correlated directly with the duration of the critical period of visual development: Huang et al. (1999) found that transgenic mice that overexpressed BDNF exhibited accelerated visual development and early closure of the critical period.

Because learning that occurs during sensitive periods lays the foundations for future learning processes (Knudsen, 2004), BDNF can be considered to play a role in memory formation. Research on the biochemical pathways leading to memory formation has concentrated on working memory through studies that addressed, for example, the Morris water maze, conditioned taste aversion, and the chick passive avoidance memory (Johnston et al., 1999; Hall et al., 2000; Johnston and Rose, 2001; 
Alonso et al., 2002; Vaynman et al., 2004). Nevertheless, the question of similarities between mechanisms involved in long-term storage of information in different species and in different areas in the brain is a fundamental issue that should be addressed. In the present study, we looked at the biochemical pathway underlying plasticity in a different neuroanatomical location (i.e., hypothalamic-related plasticity). The biochemical mechanism underlying the establishment of temperature control has not been investigated in other animals, but the present findings can be compared with those regarding other memory systems: in both passive avoidance memory in chicks (Johnston et al., 1999; Johnston and Rose, 2001) and in the Morris water maze (Linnarsson et al., 1997; Croll et al., 1999; Alonso et al., 2002), it has been shown that BDNF is the dominant neurotrophin. Furthermore, even in long-term potentiation, which serves as a model for memory storage, BDNF induces long-term potentiation (Castern et al., 1993; Kang and Schuman, 1995). We found that there was no change in NT-3 and NGF expression during thermal conditioning, and these findings correlate with previous findings, for example, that antisense inhibition of NT-3 and NGF did not affect passive avoidance learning ability in chicks, whereas BDNF-antisense inhibition disrupted memory formation (Johnston and Rose, 2001).

The center for regulation of body temperature control in both avians and mammals is neuroanatomically located in the $\mathrm{PO} / \mathrm{AH}$, which monitors local temperature changes and integrates temperature information received from the periphery. It is well established that the temperature set point can be altered during two stages of life: it can be manipulated during development by thermal conditioning in the critical period of sensory development (Tzschentke and Basta, 2002), and it can also be altered by a slow process of acclimation throughout life (Kelso and Boulant, 1982; Boulant and Dean, 1986; Griffin et al., 1996; Boulant, 1998; Pierau et al., 1998). In both cases, there is a plastic change in the ratio between hot- and cold-sensitive cells in the PO/AH. In the present study, we examined whether the induction of BDNF was a mechanism common to both of these processes and found that it was induced only in conditioning during the critical period of thermal establishment and not in acclimation in a more mature chick. Nevertheless, we cannot conclude that BDNF is not involved in acclimation because the expression level was evaluated after $9 \mathrm{~d}$ of acclimation and induction could have occurred earlier. Another possibility is that, because acclimation is a slow process, the induction of BDNF could have been small and below our detection threshold.

To knock-down BDNF in the frontal hypothalamus while causing minimal structural damage to the brain, antisense was administered to the third ventricle. Inhibition in the hypothalamus was very effective, reaching up to $90 \%$ inhibition $6 \mathrm{~h}$ after injection; nevertheless, the possibility that the phenotype observed as a result of BDNF inhibition could be attributed to BDNF inhibition in other parts of the brain cannot be completely ruled out. It has been shown previously by our group that partial knock-down of potassium channels was sufficient to impair memory acquisition (Meiri et al., 1997, 1998; Lamensdorf et al., 1999).

BDNF inhibition during the critical period of thermal control establishment caused changes in the thermal balance and in the chicks' reaction to extreme environmental conditions later in life, many days after the effect of BDNF inhibition stopped. We assume that the lack of BDNF attenuated the modulation of the thermal control-related network. Environmental temperature of $30^{\circ} \mathrm{C} 1$ week after antisense treatment caused a reduction in the chicks' naive body temperature. Because the normal body temperature of chicks in this age is $\sim 41^{\circ} \mathrm{C}$, the difference between environmental temperature and body temperature was large and therefore the antisense injected chicks could not maintain their body temperature. Furthermore, when heat challenged later in life, chicks in which BDNF had been inhibited and they received either low or high thermal input (conditioned or nonconditioned chicks) during the critical period could not maintain their body temperature, and their nutrition abilities were reduced. When extrasensory input is applied during a critical period, the sensory capabilities should be improved, but it was found that, under heat challenge, conditioned, antisense-injected chicks were more impaired than nonconditioned chicks in their capability to maintain their body temperature. A possible explanation of these findings is that the damage to the temperature network organization might be enhanced by the application of greater sensory input. BDNF inhibition did not affect the body reaction through a change in the secretion of corticosterone. Similar to previously reported results in noninjected chicks (Labunskay and Meiri, 2006), under heat challenge, the plasma corticosterone concentration of antisense-injected chicks that were conditioned did not increase, whereas that of unconditioned chicks increased significantly $3 \mathrm{~h}$ after the beginning of conditioning (data not shown).

The working hypothesis underlying the present study was that changes in neuronal connections and network modification are fundamental to the establishment of thermal control. In previous work that used differential display screening, R-Ras3 was correlated with the biochemical pathway underlying the establishment of thermal control, through the activation of Jun (Labunskay and Meiri, 2006), and the present findings indicate that BDNF expression is a critical step in thermal control establishment. Together, these results might point to the role of the BDNF-RRas3-Jun pathway during the critical period of thermalexperience-dependent developmental plasticity. It is tempting to conclude from these results that induction of growth signaling during the establishment of thermal control can probably account for the changes in cellular properties in the $\mathrm{PO} / \mathrm{AH}$ that lead to changes in the thermal set point.

\section{References}

Alonso M, Vianna MR, Depino AM, Mello e Souza T, Pereira P, Szapiro G, Viola H, Pitossi F, Izquierdo I, Medina JH (2002) BDNF-triggered events in the rat hippocampus are required for both short- and long-term memory formation. Hippocampus 12:551-560.

Berardi N, Pizzorusso T, Maffei L (2000) Critical periods during sensory development. Curr Opin Neurobiol 10:138-145.

Boettiger CA, Doupe AJ (2001) Developmentally restricted synaptic plasticity in a songbird nucleus required for song learning. Neuron 13:809-818.

Boulant JA (1998) Cellular mechanisms of temperature sensitivity in hypothalamic neurons. Prog Brain Res 115:1-8.

Boulant JA, Dean JB (1986) Temperature receptors in the central nervous system. Annu Rev Physiol 48:639-654.

Castern E, Pitkamen M, Sirvio J, Parasadanian A, Lindholm D, Thoenen H, Riekkinen PJ (1993) The induction of LTP increases BDNF and NGF mRNA but decreases NT-3 mRNA in the dentate gyrus. NeuroReport 4:895-898.

Croll SD, Suri C, Compton DL, Simmons MV, Yancopoulos GD, Lindsay RM, Wiegand SJ, Rudge JS, Scharfman HE (1999) Brain-derived neurotrophic factor transgenic mice exhibit passive avoidance deficits, increased seizure severity and in vitro hyperexcitability in the hippocampus and entorhinal cortex. Neuroscience 93:1491-1506.

Gluske RAW, Kim DS, Castern E, Thoenen H, Singer W (1996) Brainderived neurotrophic factor reserves experience-dependent synaptic modulation in kitten visual cortex. Eur J Neurosci 8:1554-1559.

Griffin JD, Kapel ML, Chow AR, Boulant JA (1996) Cellular mechanisms for the thermosensitivity in the rat hypothalamus J Physiol (Lond) 492:231-242. 
Hall J, Thomas KL, Everitt BJ (2000) Rapid and selective induction of BDNF expression in the hippocampus during contextual learning. Nat Neurosci 3:533-535.

Hensch TK (2004) Critical period regulation. Annu Rev Neurosci 27:549-579.

Horowitz M (1998) Do cellular heat acclimation responses modulate central thermoregulatory activity. News Physiol Sci 13:218-225.

Huang ZJ, Kirkwood A, Pizzorusso T, Porciatti V, Morales B, Bear MF, Maffei L, Tonegawa S (1999) BDNF regulates the maturation of inhibition and the critical period of plasticity in mouse visual cortex. Cell 17:739-755.

Johnston AN, Rose SP (2001) Memory consolidation in day-old chicks requires BDNF but not NGF or NT-3; an antisense study. Mol Brain Res $88: 26-36$.

Johnston AN, Clements MP, Rose SPR (1999) Role of brain-derived neurotrophic factor and presynaptic proteins in passive avoidance learning in day-old domestic chicks. Neuroscience 88:1033-1042.

Kang HJ, Schuman EM (1995) Long lasting neurotrophin-induced enhancement of synaptic transmission in the adult hippocampus. Science 267:1658-1662.

Kelso SR, Boulant JA (1982) Effect of synaptic blockade on thermosensitive neurons in hypothalamic tissue slices. Am J Physiol 243:R480-R490.

Kesslak JP, So V, Choi J, Cotman CW, Gomez-Pinilla F (1998) Learning upregulates brain-derived neurotrophic factor mRNA: a mechanism to facilitate encoding and circuit maintenance? Behav Neurosci 112:1212-1219.

Knudsen EI (2004) Sensitive periods in the development of the brain and behavior. J Cogn Neurosci 16:1412-1425.

Kramer R, Cohen D (2004) Functional genomics to new drug targets. Nat Rev Drug Discov 3:965-972.

Kuenzel WJ, Masson M (1988) A stereotaxic atlas of the brain of the chick. London: Johns Hopkins UP.

Labunskay G, Meiri N (2006) R-Ras3/(M-Ras) is involved in thermal adaptation in the critical period of thermal control establishment. J Neurobiol $66: 56-70$.

Lamensdorf I, Meiri N, Harvey-White J, Jacobowitz DM, Kopin IJ (1999) Kir6.2 oligoantisense administered into the globus pallidus reduces apomorphine-induced turning in 6-OHDA hemiparkinsonian rats. Brain Res 818:275-284.

Linnarsson S, Bjuorklund A, Ernfors P (1997) Learning deficit in BDNF mutant mice. Eur J Neurosci 9:2581-2587.
Ma YL, Wang HL, Wu HC, Wei CL, Lee EHY (1998) Brain-derived neurotrophic factor antisense oligonucleotide impairs memory retention and inhibits long-term potentiation in rats. Neuroscience 82:957-967.

Matsushima T, Izawa E, Aoki N, Yanagihara S (2003) The mind through chick eyes: memory, cognition and anticipation. Zool Sci 20:395-408.

Meiri N, Ghelardini C, Tesco G, Galeotti N, Dahl D, Tomsic D, Cavallaro S, Quattrone A, Capaccioli S, Bartolini A, Alkon DL (1997) Reversible antisense inhibition of Shaker-like kv1.1 potassium channel expression impairs associative memory in mouse and rat. Proc Natl Acad Sci USA 94:4430-4434.

Meiri N, Sun MK, Segal Z, Alkon DL (1998) Memory and LTP dissociated: normal spatial memory despite CA1 LTP elimination with Kv1.4 antisense. Proc Natl Acad Sci USA 95:15037-15042.

Mizuno M, Yamada K, Olariu A, Nawa H, Nabehishama T (2000) Involvement of brain-derived neurotrophic factor in spatial memory formation and maintenance in radial arm maze test in rats. J Neurosci 20:2122-2130.

Modrey P, Nichelman M (1992) Development of autonomic and behavioral thermoregulation in turkeys (Melegaris gallopavo). J Thermal Biol 17:287-290.

Pierau FK, Sann H, Yakimova KS, Haug P (1998) Plasticity of hypothalamic temperature-sensitive neurons. Prog Brain Res 115:63-84.

Robinson ESJ, Nutt DJ, Jackson HC, Hudson AL (1997) Antisense oligonucleotides in psychopharmacology and behavior: promises and pitfalls. J Psychopharmacol 11:259-269.

Tokuyama W, Okuno H, Hashimoto T, Xin Li Y, Miyashita Y (2000) BDNF upregulation during declarative memory formation in monkey inferior temporal cortex. Nat Neurosci 3:1134-1142.

Tzschentke B, Basta D (2000) Development of hypothalamic neuronal thermosensitivity in birds during the prenatal period. J Thermal Biol 25:119-123.

Tzschentke B, Basta D (2002) Early development of neuronal hypothalamic thermosensitivity in birds: influence of epigenetic temperature adaptation. Comp Biochem Physiol A Mol Integr Physiol 131:825-832.

Vaynman S, Ying Z, Gomez-Pinilla F (2004) Hippocampal BDNF mediates the efficacy of exercise on synaptic plasticity and cognition. Eur J Neurosci 20:2580-2590.

Yahav S, McMurtry JP (2001) Thermotolerance acquisition in broiler chickens by temperature conditioning early in life - the effect of timing and ambient temperature. Poult Sci 80:1662-1666. 